\title{
The role of Campylobacter spp. in chronic enteropathy in dogs
}

\section{Milos Vavra ${ }^{1}$, Gabriela Borilova ${ }^{2,3}$, Michal Fusek ${ }^{4}$, Vojtech Gabriel ${ }^{1}$, Vaclav Ceplecha ${ }^{1}$, Misa Skoric ${ }^{5}$, Michal Crha ${ }^{1}$}

${ }^{1}$ University of Veterinary and Pharmaceutical Sciences Brno, Faculty of Veterinary Medicine, Small Animal Clinic, Brno, Czech Republic

${ }^{2}$ University of Veterinary and Pharmaceutical Sciences Brno, Faculty of Veterinary Hygiene and Ecology, Department of Meat Hygiene and Technology, Brno, Czech Republic

${ }^{3}$ CEITEC - Central European Institute of Technology, University of Veterinary and Pharmaceutical Sciences Brno, Czech Republic

${ }^{4}$ Brno University of Technology, Faculty of Electrical Engineering and Communication, Department of Mathematics, Brno, Czech Republic

${ }^{5}$ University of Veterinary and Pharmaceutical Sciences Brno, Faculty of Veterinary Medicine, Department of Pathological Morphology and Parasitology, Brno, Czech Republic

Received May 16, 2019

Accepted June 13, 2019

\begin{abstract}
The aim of the study was to identify Campylobacter species in a group of patients with chronic gastrointestinal problems and to investigate the relationship between the presence of Campylobacter spp. in stool samples and as well as the severity of chronic enteropathy. Twentysix dogs with chronic gastrointestinal problems were included in the prospective study. Each research subject had their stomach, duodenum, ileum, and colon examined endoscopically. A histopathological examination of the obtained biopsy samples was then performed, excluding other potential diseases. Stool samples were collected and then examined for the presence of Campylobacter spp. To evaluate the relationship between Campylobacter spp. occurrence and the intensity of chronic enteropathy, patients were divided into two groups; animals in the first group presented with no to mild inflammation whereas research subjects in the second group suffered from moderate to severe inflammation. Subsequently, the patients were divided based on positive or negative test results for Campylobacter spp. cultures. No significant relationship between the presence of Campylobacter spp. in stool samples and chronic enteropathy was found. In contrast to other previously published papers, our study showed a lower occurrence of Campylobacter upsaliensis.
\end{abstract}

Stomach, duodenum, ileum, colon, dog, endoscopy

Campylobacter is a gram-negative curved or spiral bacteria from the Campylobacteriaceae family (Rosenberg et al. 2014). Recent studies suggest that Campylobacter concisus participates in the progression of the inflammatory bowel disease (IBD) in humans (Liu et al. 2018). Animals are hosts of Campylobacter jejuni (C. jejuni) and Campylobacter coli (C. coli), which have a zoonotic potential through contaminated food and water (Galanis 2007).

In some studies, Campylobacter upsaliensis (C. upsaliensis) was found to be the most common Campylobacter in dogs (Sandberg et al. 2002; Koene et al. 2004; Hald et al. 2004; Rossi et al. 2008); other researchers suggest $C$. jejuni as the most frequent type (Hald and Madsen 1997; López et al. 2002; Workman et al. 2005; Tsai et al. 2007). A relationship between diarrhoea and Campylobacter infection has not been confirmed in dogs (Sandberg et al. 2002; Workman et al. 2005; Acke et al. 2006). It has been implied that this organism can be commensal (Engvall et al. 2003). Nevertheless, some other studies describe a correlation between the infection and clinical signs (Guest et al. 2007). No link was proven to exist between the health status of a dog infected with $C$. upsaliensis and its breed, size, sex, vaccination status, or gonad presence (Wieland et al. 2005; Acke et al. 2006; Guest et al. 2007). 
Homeostasis of the gastrointestinal tract is secured by the relationship between the reaction to pathogens with commensal bacteria and other non-offensive antigens in the intestinal lumen (Luckschander et al. 2010). This tolerance is likely based on the presence of an antigen in context with other danger signals (Ettinger and Feldman 2005; Steiner 2008). Mucosal tolerance is also based on pattern recognition receptors which can detect bacteria and react accordingly as a result of patterns associated with pathogens or natural microflora (Burgener et al. 2008).

The probable cause of chronic enteropathy (CE) in dogs is a loss of tolerance to antigens contained e.g., in food or intestinal microflora (German et al. 2003; Luckschander et al. 2006; Kleinschmidt et al. 2007). Damage to the intestinal barrier by any means (bacterial, chemical, physical) can lead to further exposure to antigens (Greger et al. 2006). This process is amplified by lower lymphocyte apoptosis (Dandrieux et al. 2008).

The most common signs of CE include weight loss, recurrent vomiting, or diarrhoea (Kleinschmidt et al. 2007). Other clinical signs can be ascites (in relation to hypoalbuminaemia) or mucous paleness in the case of gastrointestinal (GI) bleeding (German et al. 2003; Sturgess 2005).

Biopsies of the GI tract are necessary for differentiating the types of inflammation in the intestinal mucosa (Rychlik et al. 2007). It is often very hard to interpret these histological findings conclusively (Cave 2003; Schreiner et al. 2008; Steiner 2008).

This study was performed to describe the occurrence of Campylobacter spp. in a population of dogs suffering from chronic enteropathy. This could help in the understanding of the pathogenesis and a successful treatment of this condition.

\section{Materials and Methods}

Research subjects of this study were dogs brought to the Small Animal Clinic of the University of Veterinary and Pharmaceutical Sciences Brno. These patients suffered from chronic gastrointestinal problems (i.e. they showed one or more signs thereof - anorexia, vomiting, diarrhoea, flatulence, borborygm, haematochesia, or weight loss). These symptoms had to be present for more than 3 weeks. Twenty-six dogs were included in the study, of which 16 were females and 10 were males. Altogether, they belonged to 15 different breeds (4 Yorkshire terriers, 3 German Shepherds, 2 Maltese dogs, 2 Weimaraners, 2 Chihuahuas, 1 Slovak Cuvac, 1 Chinese Crested dog, 1 Hungarian Short-Haired Pointer, 1 American Staffordshire Terrier, 1 Hanover Hound, 1 English Springer Spaniel, 1 Shetland Sheepdog, 1 Tibetan Mastiff, 1 Dachshund, 1 Labrador Retriever, 3 mixed breed dogs). These dogs were aged from 22 months to 12 years (mean age was 5.79 years; median age was 5.38 years).

A laboratory examination for the presence of Campylobacter spp. in stool samples was performed using standardized methodology (EN ISO 10272-1) in the Microbiology Laboratory of the Department of Meat Hygiene and Technology, Faculty of Veterinary Hygiene and Ecology of the University of Veterinary and Pharmaceutical Sciences Brno. Species identification was carried out by molecular biology methods. Extraction of DNA from bacterial cultures by the DNeasy ${ }^{\circledR}$ Tissue kit (Qiagen, Germany) method was performed according to the manufacturer's protocol. Species identification of thermophilic Campylobacter spp. was performed by the PCR-RFLP analysis of the variable part of the gene 23S rRNA (Fermer and Engvall 1999) and co-identified by sequencing analysis of the Hsp60 gene (previously known as cpn60 gene) according to Hill et al. (2006), which enables to identify both thermophilic and non-thermophilic Campylobacter spp.

In order to exclude other diseases, laboratory examinations (complete blood count, biochemical blood tests and parasitology examination of stool) were performed as well.

The patients then underwent endoscopic examination of the gastrointestinal tract; they were anaesthetized, intubated, and placed in left lateral recumbency. Flexible endoscopes (Olympus Evis Exera II - gastrovideoscope type GIF-XP180N and colonoscope type CF-Q165L) were used for the examination. An endoscopy score from 0 to 3 based on the visual observation of the mucosal condition was assigned. A score of 0 was set to be a physiologic mucosal finding. The presence of lightly fragile or slightly erythematous mucosa carried a score of 1 . A score of 2 was assigned to a fragile hyperaemic mucosa and score of 3 was then designated as a highly fragile mucosa with ulcerations or a cobblestone presentation (Allenspach et al. 2007). Biopsy samples were taken using flexible biopsy forceps for work channels of $2 \mathrm{~mm}$ and a $2.8 \mathrm{~mm}$ diameter depending on the endoscope used. At least 12 biopsy samples were collected from the stomach ( 3 samples from each area - fundus, curvatura major, incisura angularis, and antrum pylori). The number of duodenal biopsy samples varied based on the size of the patient and the length of the 
endoscope used. In each case, at least 10 samples were collected. During ileoscopy and colonoscopy, at least 10 samples were collected from the whole length of the intestine. More samples were collected in case local changes were spotted in various parts of the examined organs; in such instances, the material for biopsy was collected from macroscopically changed tissues. All samples were fixed in a $10 \%$ buffered formaldehyde solution.

A histopathological examination was performed in the Laboratory of the Department of Pathological Morphology and Parasitology, Faculty of Veterinary Medicine, University of Veterinary and Pharmaceutical Sciences Brno. All samples were examined by a single pathologist with no information about the history, clinical signs or endoscopic findings. A histologic lesion severity system from 0 to 3 was established, with 0 being a normal histopathologic finding, 1 defined as a mild CE (with no disruption of mucosa present, also no fibrosis of the lamina propria observed). A score of 3 was defined as a severe CE (with distortion of mucosal architecture as necrosis, large-scale ulceration, fibrosis of the lamina propria). A score of 2 was characterized as a moderate $\mathrm{CE}$ and was assigned to histopathologic findings in between the previous CE groups (Jergens et al. 2003).

The patients were divided into four groups based on the intensity of the inflammation found during endoscopy and the histopathological examination. In order to evaluate the data, a statistical analysis of contingency tables (Agresti 2012) was used. All the calculations described below were carried out in Matlab (version R2017a). The null hypothesis that there is no significant relationship between the presence of Campylobacter in faeces and the degree of nonspecific intestinal inflammation of individual organs was set against the alternative that such a relationship exists at the significance level of 0.05 . Since some frequencies in the contingency tables were too small which would lead to a violation of the model assumptions, it was necessary to merge the individual levels of inflammation into two groups. For the purpose of testing the null hypothesis, Fisher's exact test was used.

\section{Results}

Microbiological tests confirmed Campylobacter spp. in 16 patients $(61.5 \%)$ out of 26 examined dogs. Campylobacter coli was found in $5 \operatorname{dogs}(19.2 \%)$, C. jejuni was identified in 5 dogs (19.2\%), C. upsaliensis was confirmed in 1 case (3.8\%), and in 5 dogs $(19.2 \%)$ the species could not be identified.

Another aim of this study was to evaluate the relationship between Campylobacter spp. in the faeces, and the degree of non-specific inflammatory disease in the stomach, small intestine, ileum and colon of the subjects. The degree of inflammation was investigated using endoscopy as well as histopathological examination. Table 1 shows the difference between endoscopic and histopathological examination with regard to the degree of inflammation. The histology, which is generally considered to be a more reliable method, proves higher degrees of inflammation. Results of the endoscopy depend, to a great extent, on the veterinarian's subjective assessment which can differ from the more accurate histopathological examination. On average, the endoscopies showed milder degrees of inflammation than the histology eventually revealed.

Table 1. Contingency tables of the occurrence of Campylobacter spp. (positive/negative) in relation to the degree of inflammation of the GI tract in dogs; 0 - healthy, 1 - mild inflammation, 2 - moderate inflammation, 3 - severe inflammation.

\begin{tabular}{llcccccccc}
\hline & \multicolumn{4}{c}{ Endoscopy } & \multicolumn{5}{c}{ Histology } \\
\hline Degree of inflammation & 0 & 1 & 2 & 3 & 0 & 1 & 2 & 3 \\
\hline \multirow{2}{*}{ Stomach } & Negative & 1 & 7 & 2 & 0 & 0 & 9 & 1 & 0 \\
\multirow{3}{*}{ Duodenum } & Positive & 1 & 12 & 2 & 1 & 0 & 12 & 3 & 1 \\
& Negative & 1 & 3 & 5 & 1 & 0 & 1 & 8 & 1 \\
\multirow{3}{*}{ Ileum } & Positive & 1 & 8 & 5 & 2 & 1 & 5 & 8 & 2 \\
\multirow{3}{*}{ Colon } & Negative & 1 & 2 & 0 & 0 & 0 & 1 & 2 & 0 \\
& Positive & 1 & 5 & 2 & 0 & 0 & 4 & 4 & 0 \\
& Negative & 5 & 1 & 0 & 0 & 0 & 2 & 4 & 0 \\
& Positive & 5 & 3 & 2 & 0 & 0 & 3 & 5 & 2 \\
\hline
\end{tabular}


As mentioned above, in order to carry out the statistical analysis, particular degrees of inflammation had to be merged into two groups. The first group included healthy dogs or individuals with a mild form of inflammation (physiological reaction to dietary indiscretion, dietary mistakes, consumption of food contaminated by bacteria, organism-level stress responses). The second group contained patients with moderate to severe inflammation (i.e. with clinical manifestation of the disease). It was discovered that Fisher's test does not reject the null hypothesis of independence in all cases (Table 2). All the calculated $P$-values were significantly higher than the defined significance level of 0.05 . The lowest $P$-value $(0.19)$ was obtained in case of the histopathological examination of the small intestine. The relationship between Campylobacter spp. occurrence in faeces and the degree of chronic enteropathy of particular organs was not confirmed.

Table 2. Contingency tables (with merged inflammation levels) of the occurrence of Campylobacter spp. (positive/negative) in relation to the degree of inflammation of the GI tract in dogs $(0$ - healthy, 1 - mild inflammation, 2 - moderate inflammation, 3 - severe inflammation); $P$-values of Fisher's exact test are included.

\begin{tabular}{|c|c|c|c|c|c|c|c|}
\hline & & \multicolumn{3}{|c|}{ Endoscopy } & \multicolumn{3}{|c|}{ Histology } \\
\hline \multicolumn{2}{|c|}{ Degree of inflammation } & $0-1$ & $2-3$ & $P$ & $0-1$ & $2-3$ & $P$ \\
\hline \multirow{2}{*}{ Stomach } & Negative & 8 & 2 & 1.00 & 9 & 1 & 0.62 \\
\hline & Positive & 13 & 3 & & 12 & 4 & \\
\hline \multirow{2}{*}{ Duodenum } & Negative & 4 & 6 & 0.69 & 1 & 9 & 0.19 \\
\hline & Positive & 9 & 7 & & 6 & 10 & \\
\hline \multirow{2}{*}{ Ileum } & Negative & 3 & 0 & 1.00 & 1 & 2 & 1.00 \\
\hline & Positive & 6 & 2 & & 4 & 4 & \\
\hline \multirow{2}{*}{ Colon } & Negative & 6 & 0 & 0.50 & 2 & 4 & 1.00 \\
\hline & Positive & 8 & 2 & & 3 & 7 & \\
\hline
\end{tabular}

\section{Discussion}

In comparison with the study by Králová (2012) who found the prevalence of Campylobacter spp. in dogs to be $87.5 \%$, this study showed only $61.5 \%$. Figura (1991) reported a $13 \%$ prevalence of Campyblobacter jejuni in dogs, whereas Králová (2012) found it to be $66.9 \%$. The prevalence of $\mathrm{C}$. jejuni was found to be $19.2 \%$ in our study. We found a lower prevalence of $C$. upsaliensis. In our group, only $3.8 \%$ of samples were positive, compared to $20.9 \%$ of cases in the study by Králová (2012). Figura (1991) reported the prevalence of C. upsaliensis in $27 \%$ of cases. Moreover, Sandstedt et al. (1983) found $64 \%$ of samples positive (63 samples out of 98 were C. upsaliensis positive). This may have been caused by the absence of younger patients in our research group. Hald et al. (2004) states that the incidence of Campylobacter spp. (especially of C. upsaliensis) culminates at 13 to 15 months of age. On the other hand, we found the occurrence of $C$. coli to be more frequent than Králová (2012) presented (19.2\% vs. $7.4 \%)$. In 5 of the patients (19.2\%), the exact Campylobacter species could not be identified. In the study by Králová (2012), this happened in $4.8 \%$ of cases. None of the patients suffered from a Campylobacter spp. simultaneous infection. Campylobacter concisus is mentioned as being connected in human medicine with some forms of IBD (Liu et al. 2018); in this study, it was not detected in any patients.

In our study, $68.7 \%$ of female dogs and $50 \%$ of male dogs proved to be Campylobacter spp. positive. Králová (2012) found $84.2 \%$ of females and $90.8 \%$ of males positive in her study.

The amount of different diagnostic methods of Campylobacter spp. can lead to variation in results. The number of patients was limited, as only 26 patients were examined in this 
study. In order to put these findings into context, Figura's study (1991) was used. In his study, only 30 dogs were involved.

It might be useful to conduct an extensive study with a focus on the prevalence of $C$. upsaliensis among healthy dogs. Furthermore, the relationship of $C$. upsaliensis and CE should be examined thoroughly given the different percentage of its presence in our results and among subjects of other studies (Sandstedt et al. 1983; Figura 1991). Recent study detected a difference in the bacterial microflora in dogs with $\mathrm{CE}$ and in healthy dogs (Xenoulis et al. 2008). This research implies a correlation between the intestinal microflora and CE in dogs. Such a difference was also observed in humans with IBD (Ott et al. 2004; Takaishi et al. 2008).

A statistical error might have occurred due to the small data file. Xenoulis et al. (2008) examined the impact of $\mathrm{CE}$ on the physiological composition of bacterial microflora in other animal species. This research showed a significantly lower prevalence of $C$. upsaliensis than other studies (Figura 1991; Králová 2012).

Breed predisposition to $\mathrm{CE}$ is suspected; e.g., immunoproliferative enteropathy is common in Basenjis, Soft-Coated Wheaten Terriers suffer more frequently from proteinlosing enteropathy, and Boxers are prone to histiocytic colitis (German et al. 2003; Steiner 2008). Several other breeds, such as the French Bulldog, Doberman, Mastiff, and Alaskan Malamute seem to be predisposed to histiocytic colitis as well (Stokes et al. 2001; Steiner 2008). As for this research none of the predisposed dog breeds were present in the study.

In conclusion, the occurrence of $C$. upsaliensis was found to be lower than other studies show; our recommendation would be to proceed with research on the C. upsaliensis presence in dogs. We did not conclude that the severity of chronic enteropathy could be connected to Campylobacter spp. being present in faeces samples. The stage of inflammation assessed using endoscopy does not correlate exactly to the histopathological results. Endoscopy connected with histopathological examination of the GI tract is of the utmost importance for the diagnosis and staging of inflammation.

\section{Conflict of interest}

The authors disclose no conflict of interest. None of the authors have any financial or personal relationships that could inappropriately influence or bias the content of the paper.

\section{Acknowledgements}

This work was supported by the project "CEITEC - Central European Institute of Technology" (CZ.1.05/1.1.00/02.0068) from the European Regional Development Fund and by the Institutional Program of Faculty of Veterinary Medicine, University of Veterinary and Pharmaceutical Sciences Brno. The authors would like to thank the editor and the anonymous reviewers for a number of good suggestions which helped to improve the manuscript.

\section{References}

Acke E, Whyte P, Jones BR, McGill K, Collins JD, Fanning S 2006 : Prevalence of thermophilic Campylobacter species in cats and dogs in two animal shelters in Ireland. Vet Rec 158: $51-54$

Agresti A 2012: Categorical Data Analysis, ${ }^{\text {rd }}$ Edition. Wiley, Gainesville, 752 p.

Allenspach K, Wieland B, Gröne A, Gaschen F 2007: Chronic enteropathies in dogs: evaluation of risk factors for negative outcome. J Vet Intern Med 21: 700-708

Burgener IA, König A, Allenspach K, Sauter SN, Boisclair J, Doherr MG, Jungi TW 2008: Upregulation of toll-like receptors in chronic enteropathies in dogs. J Vet Intern Med 22: 553-560

Cave NJ 2003: Chronic inflammatory disorders of the gastrointestinal tract of companion animals. N Z Vet J 51: $262-274$

Dandrieux JR, Bornand VF, Doherr MG, Kano R, Zurbriggen A, Burgener IA 2008: Evaluation of lymphocyte apoptosis in dogs with inflammatory bowel disease. Am J Vet Res 69: 1279-1285

Engvall EO, Brändstrom B, Andersson L, Båverud V, Trowald-Wigh G, Englund L 2003: Isolation and 
identification of thermophilic Campylobacter species in faecal samples from swedish dogs. Scand J Infect Dis 35: $713-718$

EN ISO (2006). EN ISO 10272-1. Microbiology of food and animal feeding stuffs - Horizontal method for the detection and enumeration of Campylobacter spp. - Part 1: Detection method. Prague: Czech Office for Standards, Metrology and Testing, 2006

Ettinger SJ, Feldman EC 2005: Textbook of Veterinary Internal Medicine. $6^{\text {th }}$ Rev Edition. Saunders, Missouri, $2208 \mathrm{p}$.

Fermér C, Engvall EO 1999: Specific PCR identification and differentiation of the thermophilic campylobacters, Campylobacter jejuni, C. coli, C. lari and C. upsaliensis. J Clin Microbiol 37: 3370-3373

Figura N 1991: Campylobacter spp. isolated from dog faeces. Lancet Lond Engl 338: 1403

Galanis E 2007: Campylobacter and bacterial gastroenteritis. CMAJ Can Med Assoc J 177: 570-571

German AJ, Hall EJ, Day MJ 2003: Chronic intestinal nflammation and intestinal disease in dogs. J Vet Intern Med 17: 8-20

Greger DL, Gropp F, Morel C, Sauter S, Blum JW 2006: Nuclear receptor and target gene mRNA abundance in duodenum and colon of dogs with chronic enteropathies. Domest Anim Endocrinol 31: 327-339

Guest CM, Stephen JM, Price CJ 2007: Prevalence of Campylobacter and four endoparasites in dog populations associated with hearing dogs. J Small Anim Pract 48: 632-637

Hald B, Madsen M 1997: Healthy puppies and kittens as carriers of Campylobacter spp., with special reference to Campylobacter upsaliensis. J Clin Microbiol 35: 3351-3352

Hald B, Pedersen K, Wainø M, Jørgensen JC, Madsen M 2004: Longitudinal study of the excretion patterns of thermophilic Campylobacter spp. in young pet dogs in Denmark. J Clin Microbiol 42: 2003-2012

Hill JE, Paccagnella A, Law K, Melito PL, Woodward DL, Price L, Leung AH, Ng LK, Hemmingsen SM, Goh SH 2006: Identification of Campylobacter spp. and discrimination from Helicobacter and Arcobacter spp. by direct sequencing of PCR-amplified cpn60 sequences and comparison to cpnDB, a chaperonin reference sequence database. J Med Microbiol 55: 393-399

Jergens AE, Schreiner CA, Frank DE, Niyo Y, Ahrens FE, Eckersall PD, Benson TJ, Evans R 2003: A scoring index for disease activity in canine inflammatory bowel disease. J Vet Intern Med 17: 291-297

Kleinschmidt S, Meneses F, Nolte I, Hewicker-Trautwein M 2007: Characterization of mast cell numbers and subtypes in biopsies from the gastrointestinal tract of dogs with lymphocytic-plasmacytic or eosinophilic gastroenterocolitis. Vet Immunol Immunopathol 120: 80-92

Koene MGJ, Houwers DJ, Dijkstra JR, Duim B, Wagenaar JA 2004: Simultaneous presence of multiple Campylobacter species in dogs. J Clin Microbiol 42: 819-821

Králová K 2012: Identification of thermophillic Campylobacter isolated from pets, University of Veterinary and Pharmaceutical Sciences Brno, Diploma Thesis, 68 p.

Liu F, Ma R, Wang Y, Zhang L 2018: The clinical importance of Campylobacter concisus and other human hosted Campylobacter species. Front Cell Infect Microbiol 8: 243

López CM, Giacoboni G, Agostini A, Cornero FJ, Tellechea DM, Trinidad JJ 2002: Thermotolerant Campylobacters in domestic animals in a defined population in Buenos Aires, Argentina. Prev Vet Med 55: 193-200

Luckschander N, Allenspach K, Hall J, Seibold F, Gröne A, Doherr MG, Gaschen F 2006: Perinuclear antineutrophilic cytoplasmic antibody and response to treatment in diarrheic dogs with food responsive disease or inflammatory bowel disease. J Vet Intern Med 20: 221-227

Luckschander N, Hall JA, Gaschen F, Forster U, Wenzlow N, Hermann P, Allenspach K, Dobbelaere D, Burgener IA, Welle M 2010: Activation of nuclear factor-kappaB in dogs with chronic enteropathies. Vet Immunol Immunopathol 133: 228-236

Ott SJ, Musfeldt M, Wenderoth DF, Hampe J, Brant O, Fölsch UR, Timmis KN, Schreiber S 2004: Reduction in diversity of the colonic mucosa associated bacterial microflora in patients with active inflammatory bowel disease. Gut 53: 685-693

Rosenberg E, DeLong EF, Lory S, Stackebrandt E, Thompson F (Eds) 2014: The Prokaryotes: Deltaproteobacteria and Epsilonproteobacteria. $4^{\text {th }}$ Edition. Springer, New York, 415 p.

Rossi M, Hänninen ML, Revez J, Hannula M, Zanoni RG 2008: Occurrence and species level diagnostics of Campylobacter spp., enteric Helicobacter spp. and Anaerobiospirillum spp. in healthy and diarrheic dogs and cats. Vet Microbiol 129: 304-314

Rychlik A, Nieradka R, Kander M, Depta A, Nowicki M, Sarti K 2007: Usefulness of endoscopic examination for the diagnosis of inflammatory bowel disease in the dog. Pol J Vet Sci 10: 113-118

Sandberg M, Bergsjø B, Hofshagen M, Skjerve E, Kruse H 2002: Risk factors for Campylobacter infection in Norwegian cats and dogs. Prev Vet Med 55: 241-253

Sandstedt K, Ursing J, Walder M 1983: Thermotolerant Campylobacter with no or weak catalase activity isolated from dogs. Curr Microbiol 8: 209-213

Schreiner NMS, Gaschen F, Gröne A, Sauter SN, Allenspach K 2008: Clinical signs, histology, and CD3-positive cells before and after treatment of dogs with chronic enteropathies. J Vet Intern Med 22: 1079-1083

Steiner JM 2008: Small Animal Gastroenterology. Schluetersche, Hannover, 352 p.

Stokes JE, Kruger JM, Mullaney T, Holan K, Schall W 2001: Histiocytic ulcerative colitis in three non-boxer dogs. J Am Anim Hosp Assoc 37: 461-465 
Sturgess K 2005: Diagnosis and management of idiopathic inflammatory bowel disease in dogs and cats. In Pract 27: 293-301

Takaishi H, Matsuki T, Nakazawa A, Takada T, Kado S, Asahara T, Kamada N, Sakuraba A, Yajima T, Higuchi H, Inoue N, Ogata H, Iwao Y, Nomoto K, Tanaka R, Hibi T 2008: Imbalance in intestinal microflora constitution could be involved in the pathogenesis of inflammatory bowel bisease. Int J Med Microbiol IJMM 298: 463-472

Tsai H-J, Huang H-C, Lin C-M, Lien Y-Y, Chou C-H 2007: Salmonellae and Campylobacters in household and stray dogs in Northern Taiwan. Vet Res Commun 31: 931-939

Wieland B, Regula G, Danuser J, Wittwer M, Burnens AP, Wassenaar TM, Stärk KDC 2005: Campylobacter spp. in dogs and cats in Switzerland: Risk factor analysis and molecular characterization with AFLP. J Vet Med B Infect Dis Vet Public Health 52: 183-189

Workman SN, Mathison GE, Lavoie MC 2005: Pet dogs and chicken meat as reservoirs of Campylobacter spp. in Barbados. J Clin Microbiol 43: 2642-2650

Xenoulis PG, Palculict B, Allenspach K, Steiner JM, Van House AM, Suchodolski JS 2008: Molecularphylogenetic characterization of microbial communities imbalances in the small intestine of dogs with inflammatory bowel disease. FEMS Microbiol Ecol 66: 579-589 